\title{
Quadratic Hamiltonians on non-Euclidean spaces of arbitrary constant curvature
}

\author{
James D. Biggs \\ james.biggs@strath.ac.uk
}

\begin{abstract}
This paper derives explicit solutions for Riemannian and sub-Riemannian curves on non-Euclidean spaces of arbitrary constant cross-sectional curvature. The problem is formulated in the context of an optimal control problem on a 3-D Lie group and an application of Pontryagin's maximum principle of optimal control leads to the appropriate quadratic Hamiltonian. It is shown that the regular extremals defining the necessary conditions for Riemannian and sub-Riemannian curves can each be expressed as the classical simple pendulum. The regular extremal curves are solved analytically in terms of Jacobi elliptic functions and their projection onto the underlying base space of arbitrary curvature are explicitly derived in terms of Jacobi elliptic functions and an elliptic integral.
\end{abstract}

Keywords: Riemannian curves, sub-Riemannian curves, non-Euclidean space, optimal control .

\section{INTRODUCTION}

Let $G$ denote the 3-D isometry group of a simply connected surface $S$ of constant cross-sectional curvature $\varepsilon$, and let $A_{1}, A_{2}$ and $A_{3}$ denote a basis of left-invariant vector fields in the Lie algebra $\mathfrak{g}$ of $G$ with the Lie bracket $[X, Y]=$ $X Y-Y X$ (with $X, Y \in \mathfrak{g}$ and where $X Y$ denotes matrix multiplication of $X$ and $Y$ ) defined by the commutative relations $\left[A_{1}, A_{2}\right]=\varepsilon A_{3},\left[A_{2}, A_{3}\right]=A_{1}$ and $\left[A_{1}, A_{3}\right]=-A_{2}$. Note that when $\varepsilon=1,-1,0$ we obtain the standard 3 -D matrix Lie algebras i.e. $G$ is the Special Orthogonal Group $S O(3)$ with Lie algebra $\mathfrak{s o}(3)$ when $\varepsilon=1, G$ is the Hyperbolic Group $S O(1,2)$ with Lie algebra $\mathfrak{s o}(1,2)$ when $\varepsilon=-1$, and $G$ is the Special Euclidean Group $S E(2)$ with Lie algebra $\mathfrak{s e}(2)$ when $\varepsilon=0$. In each of the standard cases the simply connected surfaces $S$ are the planar forms; the sphere $\mathbb{S}^{2}$, the hyperbola $\mathbb{H}^{2}$ and the Euclidean plane $\mathbb{R}^{2}$ with each having constant cross sectional curvature of $\varepsilon=1,-1$ and 0 respectively. In this paper we generalise to spaces of arbitrary constant cross sectional curvature, with $\varepsilon \in(-\infty, 0) \cup(0, \infty)$ ensuring that $G$ is a semi-simple Lie group while the degenerate Euclidean case $\varepsilon=0$ is considered as a limiting case. This paper considers the problem of minimizing quadratic functions of the form:

$$
J=\frac{1}{2} \int_{0}^{T} \sum_{i=1}^{n} c_{i} v_{i}^{2} d t
$$

where $i \leq n \leq 3$ and $c_{i}>0$ are constant weights and $v_{i}$ are functions on the interval $[0, T]$, satisfying the prescribed boundary conditions $g(0)=g_{0}$ and $g(T)=g_{T}$ where $g(t) \in G$ satisfies the differential constraint:

$$
\frac{d g(t)}{d t}=g(t) \sum_{i=1}^{n} A_{i} v_{i}
$$

This class of problem is associated with Riemannian geometry when $n=3$ where the metric (the integrand of equation (1)) is a positive definite quadratic form defined on the entire Lie algebra. If the metric is defined only partially on the Lie algebra $(n<3)$ the problem is a sub-Riemannian one [1], [2], [3], [4], [5]. The Riemannian problem equates to a statement of the Principle of least action for a free rigid body if $c_{1}, c_{2}, c_{3}$ are equal to the principal moments of inertia, $v_{i}$ the angular velocities and $\varepsilon=1(G \in S O(3))$ [6], [7]. In this case the Hamiltonian vector fields defining the necessary conditions for optimality are the Euler equations. In this particular case the Hamiltonian equations of the free rigid body can be reduced to the classical simple pendulum equations under a cylindrical coordinate change of variables [7]. In this paper it is shown that the necessary conditions for optimality can be reduced to the equations of the simple pendulum for a larger class of optimal control problem.

In all other cases, other than the Riemannian problem, this problem statement is associated with sub-Riemannian geometry where the integrand of (1) defines only a partial metric on the Lie algebra $(n<3)$. Sub-Riemannian curves can also be defined equivalently by the Riemannian case but with any single weight $c_{i} \rightarrow \infty$. Note that no more than one constant weight can tend to infinite as for these cases the optimal control problem is not well posed. A particular class of sub-Riemannian curves, called $\mathfrak{p}$-curves, were studied in [1] where the partial metric is defined on the vertical vector fields $\mathfrak{p}$ of the Cartan decomposition. In [1] $\mathfrak{p}$-curves are studied for the classic planar forms where their curvature $\varepsilon=-1,0,1$. The $\mathfrak{p}$-curves, in [1], correspond to the limiting case where $c_{3} \rightarrow \infty$ or equivalently setting $n=2$ in equations (1) and (2). In this paper we generalise the analysis of $\mathfrak{p}$ curves, in [1], to spaces of arbitrary constant curvature.

Another potentially interesting case is where $A_{1}$ and $A_{3}$ in (2) are controlled and $A_{2}$ is not. In other words $v_{2}=0$ in equations (1) and (2) which corresponds to the limiting Riemannian case as $c_{2} \rightarrow \infty$. In this case the differential constraint (2) can be viewed analogously to the kinematics of a wheeled robot with a nonholonomic (sliding) constraint where $v_{1}$ is the velocity in the forward direction and $v_{3}$ the angular (steering) velocity. It follows that the optimal control problem defines paths of a wheeled robot that minimises a weighted cost function of the forward velocity and the amount of required steering.

This paper solves the extremals for these Riemannian and sub-Riemannian curves in terms of Jacobi elliptic functions and shows that the equations can be reduced to the 
classical pendulum through a simple coordinate change. An integration method is then presented which generalises the procedure used to project the extremals onto $g(t) \in S O(3)$ presented in [8] to spaces of arbitrary cross-sectional curvature. This integration method is then applied to project the extremals related to Riemannian and sub-Riemannian curves onto the simply connected surface $S$. This reveals that Riemannian and sub-Riemannian curves are described by Jacobi elliptic functions and an incomplete Elliptic integral of the third kind.

\section{NECESSARY CONDITIONS FOR (SUB-) RIEMANNIAN CURVES}

An application of Pontryagin's maximum principle of optimal control (where the functions $v_{1}, v_{2}, v_{3}$ are the assumed control functions) brings us to the associated (left-invariant) Hamiltonian formalism. There is a wealth of literature on the co-ordinate free Maximum principle and in line with the geometric interpretations of this paper the interested reader should refer to [8], [5], [9], [3] for details. Each left-invariant Hamiltonian can be expressed independently of co-ordinates on $G$ as a function $f$ of the extremal curves $H=f\left(h_{1}, h_{2}, h_{3}\right)$ where $h_{1}, h_{2}, h_{3} \in \mathfrak{g}$ are the extremal curves and $h_{i}=p\left(A_{i}\right)$ with $p(\cdot)$ a scalar function which maps an element of the Lie algebra to its dual defined through the non-degenerate trace form (for $\varepsilon \in(-\infty, 0) \cup(0, \infty)$ ). Explicitly, minimising the cost function (1) subject to the constraint on the Lie algebra from (2) gives the Hamiltonian:

$$
H=\sum_{i=1}^{n} h_{i} v_{i}-\rho_{0} \frac{1}{2} \sum_{i=1}^{n} c_{i} v_{i}^{2}
$$

where $\rho_{0}=0$ for abnormal extremals and $\rho_{0}=1$ for regular extremals. Proceeding in this paper with an analysis of the regular extremals and noting that $H$ is a concave function with respect to $v_{i}$ then the optimal controls are:

$$
v_{i}^{*}=\frac{h_{i}}{c_{i}}
$$

and substituting (4) into (3) gives the optimal Hamiltonian:

$$
H=\frac{1}{2}\left(\frac{h_{1}^{2}}{c_{1}}+\frac{h_{2}^{2}}{c_{2}}+\frac{h_{3}^{2}}{c_{3}}\right)
$$

where the Hamiltonian corresponds to the Riemannian problem for arbitrary non-zero constant values of $c_{i}$ and to subRiemannian problems whenever any single constant weight $c_{i} \rightarrow \infty$. The Hamiltonian vector fields are then given by the equation $X_{H}[\cdot]=\{\cdot, H\}$ where the Poisson bracket on the dual of the Lie algebra is defined in terms of the Lie bracket as $\left\{h_{i}, h_{j}\right\}=-p\left(\left[A_{i}, A_{j}\right]\right)$. Then the Hamiltonian vector fields defining the necessary conditions for optimality are given by:

$$
\begin{aligned}
& \dot{h}_{1}=\left\{H, h_{1}\right\}=\frac{\varepsilon h_{2} h_{3}}{c_{2}}-\frac{h_{2} h_{3}}{c_{3}} \\
& \dot{h}_{2}=\left\{H, h_{2}\right\}=\frac{h_{1} h_{3}}{c_{3}}-\frac{\varepsilon h_{1} h_{3}}{c_{1}} \\
& \dot{h}_{3}=\left\{H, h_{3}\right\}=\frac{h_{1} h_{2}}{c_{2}}-\frac{h_{1} h_{2}}{c_{1}}
\end{aligned}
$$

It is easily verified that the limiting cases of the Hamiltonian vector fields as any single $c_{i} \rightarrow \infty$, correspond to the limiting cases of the Hamiltonian function, that is, the equations are well behaved. For example, as $c_{3} \rightarrow \infty$ the Hamiltonian (5) yields the Hamiltonian of general $\mathfrak{p}$-curves and (6) to the corresponding vector fields defining the necessary conditions for the existence of $\mathfrak{p}$-curves. In addition, it is easily shown that the function:

$$
M=h_{1}^{2}+h_{2}^{2}+\varepsilon h_{3}^{2}
$$

is a Casimir function for (6) i.e. $\{H, M\}=0$. Furthermore, the intersection of these functions (that implicitly define surfaces) (7) and (5) geometrically define the extremal curves [8]. In particular they are the intersection of an Ellipsoid (Riemannian Case) or elliptic cylinder (sub-Riemannian case) with an ellipsoid for $\varepsilon>0$ or a hyperbola for $\varepsilon<0$. It is also well known that the smooth intersection of any two quadric hypersurfaces in projective three space define an elliptic curve [10] which can be parameterised by elliptic functions. This gives us an indication to the form the analytic solution the extremal solutions will take.

Lemma 1: The real extremal curves associated with Riemannian and sub-Riemannian curves on 2-D simply connected surfaces of constant cross sectional curvature are described by the equation of the mathematical pendulum of arbitrary length.

Proof:

define the constants

$$
\lambda_{1}=\left(\frac{\varepsilon c_{3}-c_{2}}{c_{2} c_{3}}\right)^{2}, \lambda_{2}=\left(\frac{c_{1}-\varepsilon c_{3}}{c_{1} c_{3}}\right)^{2}, \lambda_{3}=\left(\frac{c_{1}-c_{2}}{c_{1} c_{2}}\right)^{2},
$$

then (6) can be expressed as:

$$
\left(\dot{h}_{1}\right)^{2}=\lambda_{1} h_{2}^{2} h_{3}^{2}, \quad\left(\dot{h}_{2}\right)^{2}=\lambda_{2} h_{1}^{2} h_{3}^{2}, \quad\left(\dot{h}_{3}\right)^{2}=\lambda_{3} h_{1}^{2} h_{2}^{2},
$$

using equations (5) and (7) we can write:

$$
\begin{aligned}
h_{2}^{2} & =\frac{c_{2}}{\varepsilon c_{3}-c_{2}}\left(2 c_{3} H \varepsilon+h_{1}^{2}-\frac{\left(c_{1}-\varepsilon c_{3}\right) h_{1}^{2}}{c_{1}}-M\right), \\
h_{3}^{2} & =\frac{c_{3}}{c_{2}-\varepsilon c_{3}}\left(2 c_{2} H+h_{1}^{2}-\frac{c_{2} h_{1}^{2}}{c_{1}}-M\right), \\
h_{1}^{2} & =\frac{c_{1}}{\varepsilon c_{3}-c_{1}}\left(2 c_{3} H \varepsilon+h_{2}^{2}-\frac{\varepsilon c_{3} h_{2}^{2}}{c_{2}}-M\right), \\
h_{3}^{2} & =\frac{c_{3}}{c_{1}-\varepsilon c_{3}}\left(2 c_{1} H+h_{2}^{2}-\frac{c_{1} h_{2}^{2}}{c_{2}}-M\right), \\
h_{1}^{2} & =\frac{c_{1}}{c_{2}-c_{1}}\left(2 c_{2} H-\frac{c_{2} h_{3}^{2}}{c_{3}}-M+\varepsilon h_{3}^{2}\right), \\
h_{2}^{2} & =\frac{c_{2}}{c_{1}-c_{2}}\left(2 c_{1} H-\frac{c_{1} h_{3}^{2}}{c_{3}}-M+\varepsilon h_{3}^{2}\right),
\end{aligned}
$$

and again the expressions for the sub-Riemannian case are the limits of these equations as any single $c_{i} \rightarrow \infty$. For example as $c_{3} \rightarrow \infty$ equation (10) become:

$$
\begin{aligned}
h_{2}^{2} & =2 c_{2} H-c_{2} \frac{h_{1}^{2}}{c_{1}} \\
h_{3}^{2} & =\frac{M-2 H c_{2}}{\varepsilon}+\left(\frac{c_{2}-c_{1}}{\varepsilon c_{1}}\right) h_{1}^{2} \\
h_{1}^{2} & =2 c_{1} H-c_{1} \frac{h_{2}^{2}}{c_{2}} \\
h_{3}^{2} & =\frac{M-2 H c_{1}}{\varepsilon}+\left(\frac{c_{1}-c_{2}}{\varepsilon c_{2}}\right) h_{2}^{2} \\
h_{1}^{2} & =\frac{1}{c_{2}-c_{1}}\left(2 H c_{1} c_{2}-M c_{1}+\varepsilon c_{1} h_{3}^{2}\right) \\
h_{2}^{2} & =\frac{1}{c_{1}-c_{2}}\left(2 H c_{1} c_{2}-M c_{2}+\varepsilon c_{2} h_{3}^{2}\right),
\end{aligned}
$$

then substituting in either (10) or (11) into (9) the Riemannian and sub-Riemannian curves can be expressed 
in the quadratic form:

$$
\left(\dot{h}_{i}\right)^{2}=\lambda_{i}\left(\alpha_{i} h_{i}^{2}-\beta_{i}\right)\left(k_{i} h_{i}^{2}-d_{i}\right),
$$

where $i=1,2,3$ and $\lambda_{i}$ are defined in (8) and for Riemannian curves

$$
\begin{array}{ll}
\alpha_{1}=\frac{c_{2}\left(c_{1}-c_{3} \varepsilon\right)}{c_{1}\left(c_{3} \varepsilon-c_{2}\right)}, & \beta_{1}=-\frac{c_{2}\left(2 c_{3} H \varepsilon-M\right)}{\varepsilon c_{3}-c_{2}} \\
k_{1}=\frac{c_{3}\left(c_{1}-c_{2}\right)}{c_{1}\left(c_{2}-\varepsilon c_{3}\right)}, & d_{1}=\frac{c_{3}\left(2 c_{2} H-M\right)}{\varepsilon c_{3}-c_{2}} \\
\alpha_{2}=\frac{c_{1}\left(c_{2}-\varepsilon c_{3}\right)}{c_{2}\left(\varepsilon c_{3}-c_{1}\right)}, & \beta_{2}=-\frac{c_{1}\left(2 c_{3} H \varepsilon-M\right)}{\varepsilon c_{3}-c_{1}} \\
k_{2}=\frac{c_{3}\left(c_{2}-c_{1}\right)}{c_{2}\left(c_{1}-\varepsilon c_{3}\right)}, & d_{2}=\frac{c_{3}\left(2 c_{1} H-M\right)}{\varepsilon c_{3}-c_{1}} \\
\alpha_{3}=\frac{c_{1}\left(\varepsilon c_{3}-c_{2}\right)}{c_{3}\left(c_{2}-c_{1}\right)}, & \beta_{3}=\frac{c_{1}\left(2 c_{2} H-M\right)}{c_{1}-c_{2}} \\
k_{3}=\frac{c_{2}\left(\varepsilon c_{3}-c_{1}\right)}{c_{3}\left(c_{1}-c_{2}\right)}, & d_{3}=\frac{c_{2}\left(2 c_{1} H-M\right)}{c_{2}-c_{1}}
\end{array}
$$

and for example sub-Riemannian curves when $c_{3} \rightarrow \infty$ are:

$$
\begin{aligned}
& \alpha_{i}=-\frac{c_{j}}{c_{i}}, \quad \beta_{i}=-2 c_{j} H, \quad k_{i}=\frac{c_{j}-c_{i}}{\varepsilon c_{i}}, \quad d_{i}=\frac{2 c_{j} H-M}{\varepsilon} \\
& \alpha_{3}=\frac{\varepsilon c_{1}}{c_{2}-c_{1}}, \beta_{3}=\frac{c_{1}\left(2 H c_{2}-M\right)}{c_{1}-c_{2}}, \quad k_{3}=\frac{\varepsilon c_{2}}{c_{1}-c_{2}}, d_{3}=\frac{c_{2}\left(2 H c_{1}-M\right)}{c_{2}-c_{1}}
\end{aligned}
$$

where $i=1$ when $j=2$ and $i=2$ when $j=1$. Then using the change of coordinates $h_{i}=\sqrt{\frac{b_{i}}{a_{i}}} \sin \frac{\theta}{2}$ in (12) yields the equation of the mathematical pendulum:

$$
\dot{\theta}= \pm \sqrt{A+B \cos \theta}
$$

where the constants $A=\left(4 a_{i} d_{i}-2 k_{i} b_{i}\right), B=2 k_{i} b_{i}$ where $a_{i}=\lambda_{i} \alpha_{i}$ and $b_{i}=\lambda_{i} \beta_{i}$. $\square$. The recognition of the extremal curves qualitative behaviour as being determined by the mathematical pendulum enables the description of all possible qualitative behaviours of the (sub-) Riemannian curves. Setting $I=\frac{a_{i} d_{i}}{b_{i} k_{i}}$ we define the qualitative behaviours as:

Case A: $I=0$ corresponds to the stationary position analogous to the downward position of the mathematical pendulum.

Case B: $0<I<1$ corresponds to oscillatory motion analogous to a pendulum swinging back and forth.

Case C: $I=1$ corresponds to the equation of the separatrix connecting the two saddle points of the upward equilibrium position.

Case D: $I>1$ corresponds to circulating orbits where the pendulums energy is high enough to carry the pendulum over the top.

Lemma 2: The real extremal curves associated with Riemannian and sub-Riemannian curves on 2-D simply connected surfaces of arbitrary curvature for $b_{i} k_{i}<a_{i} d_{i}$ are of the analytic form:

$$
h_{i}=\sqrt{b_{i} / a_{i}} \sin \left(z_{i}\right)
$$

where:

$$
z_{i}=\operatorname{am}\left( \pm \sqrt{a_{i} d_{i}} t+\beta_{i}, \frac{b_{i} k_{i}}{a_{i} d_{i}}\right)
$$

where $\operatorname{am}(\cdot, \cdot)$ is the Jacobi amplitude function [12] and the constant $\beta_{i}=\sin ^{-1}\left(\operatorname{am}\left(\frac{\sqrt{a_{i}} h_{i}(0)}{\sqrt{b_{i}}}, \frac{b_{i} k_{i}}{a_{i} d_{i}}\right)\right)$ and for $b_{i} k_{i}>a_{i} d_{i}$ :

$$
h_{i}=\sqrt{d_{i} / k_{i}} \sin \left(z_{i}\right)
$$

where:

$$
z_{i}=\operatorname{am}\left( \pm \sqrt{b_{i} k_{i}} t+\gamma_{i}, \frac{a_{i} d_{i}}{b_{i} k_{i}}\right)
$$

where $\operatorname{am}(\cdot, \cdot)$ is the Jacobi amplitude function [12] and the constant $\gamma_{i}=\sin ^{-1}\left(\operatorname{am}\left(\frac{\sqrt{k_{i}} h_{i}(0)}{\sqrt{d_{i}}}, \frac{a_{i} d_{i}}{b_{i} k_{i}}\right)\right)$.

Proof. It is easy to verify by substitution that this solves equation (12). Note that for $b_{i} k_{i}>a_{i} d_{i}$ the Jacobi transformation is used [11].

Here we note that (16) corresponds to Case D of the classical simple pendulum and (18) corresponds to Case A. If $b_{i} k_{i}=a_{i} d_{i}$ then each solution degenerates to a hyperbolic tan function defining the heteroclinic connection of Case $\mathrm{C}$.

Theorem 1: Riemannian and sub-Riemannian curves on a simply connected surface $S$ of cross sectional curvature $\varepsilon \in(-\infty, 0) \cup(0, \infty)$ can be expressed in terms of the extremal curves $h_{1}, h_{2}, h_{3}$ as:

$$
\begin{aligned}
& x=-\frac{h_{1}}{\sqrt{K^{2}-\varepsilon h_{3}^{2}}} \cos \phi_{1}-\frac{\sqrt{\varepsilon} h_{2} h_{3}}{K \sqrt{K^{2}-\varepsilon h_{3}^{2}}} \sin \phi_{1} \\
& y=-\frac{h_{1}}{\sqrt{K^{2}-\varepsilon h_{3}^{2}}} \sin \phi_{1}+\frac{\sqrt{\varepsilon} h_{2} h_{3}}{K \sqrt{K^{2}-\varepsilon h_{3}^{2}}} \cos \phi_{1} \\
& z=-\frac{\sqrt{\varepsilon} h_{2}}{K}
\end{aligned}
$$

where $K^{2}=M$ in (7) and

$$
\dot{\phi}_{1}=\frac{K \sqrt{\varepsilon}\left(\frac{h_{1}^{2}}{c_{1}}+\frac{h_{2}^{2}}{c_{2}}\right)}{h_{1}^{2}+h_{2}^{2}}
$$

Proof: Recall that as the Hamiltonian for sub-Riemannian curves can be viewed as limits of the Riemannian case (5) it suffices to integrate the system down to $G$ using the expression for the Hamiltonian (5). It is convenient to express the equations describing the extremal curves (6) and their relationship to $g(t) \in G$ in Lax Pair form defined on the basis of the Lie algebra:

$$
\begin{aligned}
A_{1} & =\left(\begin{array}{ccc}
0 & 0 & 1 \\
0 & 0 & 0 \\
-\varepsilon & 0 & 0
\end{array}\right), \\
A_{3} & =\left(\begin{array}{ccc}
0 & -1 & 0 \\
1 & 0 & 0 \\
0 & 0 & 0
\end{array}\right)
\end{aligned}
$$

then the (sub-)Riemannian curves are defined by the equations:

$$
\frac{d L(t)}{d t}=[d H, L(t)], \quad \frac{d g(t)}{d t}=g(t) d H
$$

where $L(t)=\sum_{i=1}^{3} h_{i} A_{i}, \quad d H=\sum_{i=1}^{3} \frac{h_{i}}{c_{i}} A_{i} . \quad$ It is easy to show by differentiation that

$$
g(t) L(t) g(t)^{-1}=\text { constant }
$$

It follows that if we define the conserved quantity $\mathrm{K}^{2}=$ $h_{1}^{2}+h_{2}^{2}+\varepsilon h_{3}^{2}$ then (24) can be conjugated such that

$$
g(t) L(t) g(t)^{-1}=\sqrt{\varepsilon} K A_{3}
$$

then defining $g(t) \in G$ in the convenient form:

$$
g(t)=\exp \left(\phi_{1} A_{3}\right) \exp \left(\phi_{2} A_{2}\right) \exp \left(\phi_{3} A_{3}\right)
$$


where $\phi_{1}, \phi_{2}, \phi_{3}$ are local coordinates then

$$
L(t)=\sqrt{\varepsilon} K g(t)^{-1} A_{3} g(t)
$$

comparing with $L(t)$ gives:

$$
\left(\begin{array}{ccc}
0 & -\varepsilon h_{3} & h_{1} \\
\varepsilon h_{3} & 0 & h_{2} \\
-\varepsilon h_{1} & -\varepsilon h_{2} & 0
\end{array}\right)=\sqrt{\varepsilon} K(\hat{x}|\hat{y}| \hat{z})
$$

where $\hat{x}, \hat{y}, \hat{z}$ are the vectors

$$
\begin{aligned}
& \hat{x}=\left[\begin{array}{lll}
0 & \cos \left(\sqrt{\varepsilon} \phi_{2}\right) & \sqrt{\varepsilon} \cos \phi_{3} \sin \left(\sqrt{\varepsilon} \phi_{2}\right)
\end{array}\right]^{T} \\
& \hat{y}=\left[\begin{array}{lll}
-\cos \left(\sqrt{\varepsilon} \phi_{2}\right) & 0 & -\sqrt{\varepsilon} \sin \phi_{3} \sin \left(\sqrt{\varepsilon} \phi_{2}\right)
\end{array}\right]^{T} \\
& \hat{z}=\left[\begin{array}{lll}
-\frac{\cos \phi_{3} \sin \left(\sqrt{\varepsilon} \phi_{2}\right)}{\sqrt{\varepsilon}} & \frac{\sin \phi_{3} \sin \left(\sqrt{\varepsilon} \phi_{2}\right)}{\sqrt{\varepsilon}} & 0
\end{array}\right]^{T}
\end{aligned}
$$

which yields

$$
\begin{aligned}
& h_{1}=-K \cos \phi_{3} \sin \left(\sqrt{\varepsilon} \phi_{2}\right) \\
& h_{2}=K \sin \phi_{3} \sin \left(\sqrt{\varepsilon} \phi_{2}\right) \\
& h_{3}=\frac{K}{\sqrt{\varepsilon}} \cos \left(\sqrt{\varepsilon} \phi_{2}\right)
\end{aligned}
$$

it follows that

$$
\cos \left(\sqrt{\varepsilon} \phi_{2}\right)=\frac{\sqrt{\varepsilon} h_{3}}{K}, \quad \sin \left(\sqrt{\varepsilon} \phi_{2}\right)=\frac{\sqrt{K^{2}-\varepsilon h_{3}^{2}}}{K}
$$

and

$$
\cos \phi_{3}=-\frac{h_{1}}{\sqrt{K^{2}-\varepsilon h_{3}^{2}}}, \quad \sin \phi_{3}=\frac{h_{2}}{\sqrt{K^{2}-\varepsilon h_{3}^{2}}}
$$

these solutions will be used in conjunction with the following. First, we substitute equation (26) into $g(t)^{-1} \frac{d g(t)}{d t}=d H$ from (23) which yields:

$$
\begin{aligned}
\frac{h_{3}}{c_{3}} & =\cos \left(\sqrt{\varepsilon} \phi_{2}\right) \dot{\phi}_{1}+\dot{\phi}_{3} \\
\frac{h_{2}}{c_{2}} & =\frac{\sin \left(\sqrt{\varepsilon} \phi_{2}\right) \sin \phi_{3} \dot{\phi}_{1}}{\sqrt{\varepsilon}}+\cos \phi_{3} \dot{\phi}_{2} \\
\frac{h_{1}}{c_{1}} & =-\frac{\sin \left(\sqrt{\varepsilon} \phi_{2}\right) \cos \phi_{3} \dot{\phi}_{1}}{\sqrt{\varepsilon}}+\sin \phi_{3} \dot{\phi}_{2}
\end{aligned}
$$

which on substitution of (31) and (32) simplifies to

$$
\begin{aligned}
\frac{h_{3}}{c_{3}} & =\frac{\sqrt{\varepsilon} h_{3}}{K} \dot{\phi}_{1}+\dot{\phi}_{3} \\
\frac{h_{2}}{c_{2}} & =\frac{h_{2} \dot{\phi}_{1}}{K \sqrt{\varepsilon}}-\frac{h_{1}}{\sqrt{K^{2}-\varepsilon h_{3}^{2}}} \dot{\phi}_{2} \\
\frac{h_{1}}{c_{1}} & =\frac{h_{1} \dot{\phi}_{1}}{K \sqrt{\varepsilon}}+\frac{h_{2}}{\sqrt{K^{2}-\varepsilon h_{3}^{2}}} \dot{\phi}_{2}
\end{aligned}
$$

it follows that:

$$
\dot{\phi}_{1}=\frac{K \sqrt{\varepsilon}\left(\frac{h_{1}^{2}}{c_{1}}+\frac{h_{2}^{2}}{c_{2}}\right)}{h_{1}^{2}+h_{2}^{2}}
$$

noting that the projection of $g(t) \in G$ (26) onto $S$ given by $g(t)\left[\begin{array}{llll}1 & 0 & 0 & 0\end{array}\right]^{T}$ is:

$$
\begin{aligned}
& x=\cos \phi_{3} \cos \phi_{1}-\cos \sqrt{\varepsilon} \phi_{2} \sin \phi_{3} \sin \phi_{1} \\
& y=\cos \phi_{3} \sin \phi_{1}+\cos \sqrt{\varepsilon} \phi_{2} \sin \phi_{3} \cos \phi_{1} \\
& z=-\sqrt{\varepsilon} \sin \sqrt{\varepsilon} \phi_{2} \sin \phi_{3}
\end{aligned}
$$

then substituting (31), (32) and (35) into (36) gives (20).

Lemma 3: The solution to the integral

$$
\dot{\phi}_{1}=\frac{K \sqrt{\varepsilon}\left(\left(h_{1}^{2}\right) / c_{1}+\left(h_{2}^{2}\right) / c_{2}\right)}{h_{1}^{2}+h_{2}^{2}}
$$

with the extremal curves defined by (16) $\left(b_{3} k_{3}<a_{3} d_{3}\right)$ is

$$
\phi_{1}=\frac{\Gamma t}{\gamma}+\frac{\left(\gamma \alpha-\Gamma K^{2}\right)}{\sqrt{a_{3} d_{3}} \gamma \Gamma K^{2}} \Pi\left[\frac{\gamma}{K^{2}}, a m\left( \pm \sqrt{a_{3} d_{3}} t+\beta_{3}, \frac{b_{3} k_{3}}{a_{3} d_{3}}\right), \frac{b_{3} k_{3}}{a_{3} d_{3}}\right]
$$

where $\Pi[\cdot, \cdot, \cdot]$ is the incomplete elliptic integral [12] and $\operatorname{am}(\cdot, \cdot)$ the Jacobi amplitude function with constants:

$$
\alpha=2 H K \sqrt{\varepsilon}, \quad \Gamma=\frac{K \sqrt{\varepsilon} b_{3}}{a_{3} c_{3}}, \quad \gamma=\frac{\varepsilon b_{3}}{c_{3}}
$$

and with the extremal curves defined by (18) $\left(b_{3} k_{3}>a_{3} d_{3}\right)$ is

$$
\begin{aligned}
& \phi_{1}=\frac{\Gamma t}{\gamma}+ \\
& \frac{\left(\gamma \alpha-\Gamma K^{2}\right)}{\sqrt{b_{3} k_{3}} \gamma \Gamma K^{2}} \Pi\left[\frac{\gamma}{K^{2}}, a m\left( \pm \sqrt{k_{3} b_{3}} t+\gamma_{3}, \frac{d_{3} a_{3}}{k_{3} b_{3}}\right), \frac{d_{3} a_{3}}{k_{3} b_{3}}\right]
\end{aligned}
$$

with constants:

$$
\alpha=2 H K \sqrt{\varepsilon}, \quad \Gamma=\frac{K \sqrt{\varepsilon} d_{3}}{k_{3} c_{3}}, \quad \gamma=\frac{\varepsilon d_{3}}{c_{3}}
$$

Proof: rearranging the differential equation (37) as an integral and using the Hamiltonian (5) and Casimir function (7), $\phi_{1}$ can be expressed in terms of $h_{3}$ as:

$$
\phi_{1}=\int \frac{K \sqrt{\varepsilon}\left(2 H-h_{3}^{2} / c_{3}\right)}{K^{2}-\varepsilon h_{3}^{2}} d t
$$

then substituting $h_{3}=\sqrt{\frac{b_{3}}{a_{3}}} \mathrm{sn}\left( \pm \sqrt{a_{3} d_{3}} t+\beta_{3}, \frac{b_{3} k_{3}}{a_{3} d_{3}}\right)$ from (16) into (42) and integrating yields (38). Equation (40) is obtained in an analagous manner.

Lemma 4: Riemannian and sub-Riemannian curves on non-Euclidean spaces of constant curvature $\varepsilon \in(-\infty, 0) \cup$ $(0, \infty)$ are of the analytic form:

$$
\begin{aligned}
& x=-\frac{\sqrt{b_{1}} \sin z_{1}}{\sqrt{a_{1}} \sqrt{K^{2}-\varepsilon \frac{b_{3}}{a_{3}} \sin z_{3}}} \cos \phi_{1}-\sqrt{\frac{\varepsilon b_{2} b_{3}}{a_{2} a_{3}}} \sin z_{2} \sin z_{3} \sin \phi_{1} \\
& y=-\frac{\sqrt{b_{1}} \sin z_{1}}{\sqrt{a_{1}} \sqrt{K^{2}-\varepsilon \frac{b_{3}}{a_{3}} \sin z_{3}}} \sin \phi_{1}+\sqrt{\frac{\varepsilon b_{2} b_{3}}{a_{2} a_{3}}} \sin z_{2} \sin z_{3} \cos \phi_{1} \\
& z=-\frac{\sqrt{\varepsilon b_{2}}}{K \sqrt{a_{2}}} \sin z_{2}
\end{aligned}
$$

where $z_{i}$ is the Jacobi amplitude function (17) for $b_{i} k_{i}<a_{i} d_{i}$ and (19) for $b_{i} k_{i}>a_{i} d_{i}$ and where $\phi_{1}$ is defined by (38) for $\left(b_{3} k_{3}<a_{3} d_{3}\right)$ and (40) for $\left(b_{3} k_{3}>a_{3} d_{3}\right)$.

\section{CONCLUSION}

In this paper a closed form solution for Riemannian and sub-Riemannian curves on non-Euclidean spaces of arbitrary curvature are derived. The projection of the curves onto the base space are expressed in terms of Jacobi elliptic functions and trigonometric functions of the sum of a secular term and an incomplete elliptic integral.

\section{REFERENCES}

[1] Jurdjevic, V., 'Hamiltonian point of view of non-euclidean geometry and elliptic functions'. Systems and control letters, No. 42, pp. 25-41, 2001.

[2] do Carmo, M. P., 'Riemannian Geometry'. Mathematics: Theory and Applications, Birkhauser, Boston, 1992.

[3] Bloch, A. M., 'Nonholonomic Mechanics and Control'. SpringerVerlag,

[4] Brockett, R. W., 'Control Theory and singular Riemannian geometry'. in New Directions in Applied Mathematics (P.Hilton and G.Young, eds.), Springer-Verlag,New York,p. 11-27, 1981.

[5] Agrachev, A. A., Sachkov, Y. L. 'Control theory from a geometric viewpoint' Springer-Verlag, Berlin, 2010.

[6] Holmes, D. D.,'Geometric Mechanics, Part 1: Dynamics and Symmetry'.Imperial College Press. pp. 105-110, 2008. 
[7] Holmes, D. D., Marsden, J. E., 'The Rotor and the Pendulum'.Imperial College Press. In Symplectic Geometry and Mathematical Physics, (P. Donato and C. Duval and J. Elhadad and G. M. Tuynman, eds.), Birkhuser, Boston, pp. 189-203, 1991.

[8] Jurdjevic, V., 'Geometric Control Theory'. Advanced Studies in Mathematics, Cambridge University Press, 52, 1997.

[9] Sussmann, H. J., 'An introduction to the coordinate-free maximum principle'. In Geometry of Feedback and Optimal Control,B. Jakubczyk and W. Respondek Eds., Marcel Dekker, New York, pp. 463-557, 1997.

[10] D. Husemoller, Elliptic Curves. Springer-Verlag, New York, 2000.

[11] Olver, F., Lozier D, Boisvert, R., Clark, C., 'NIST Handbook of Mathematical Functions', Cambridge University Press, 2010.

[12] Lawden, D., 'Elliptic functions and applications'. Springer-Verlag, New York, 1989. 